\section{Terapias biológicas en el lupus eritematoso sistémico}

\author{
ERNESTO CAIROLI ${ }^{1,2}$, GERARD ESPINOSA $^{3}$, RICARD CERVERA $^{3}$
}

\section{Biological therapies in systemic lupus erythematosus}

The immunosuppressive agents used in patients with systemic lupus erythematosus (SLE) have significantly improved prognosis. However, it is necessary to develop more specific immunosuppressive treatments with less toxicity. Better understanding of the mechanisms involved in the loss of tolerance in autoimmune diseases has contributed to the development of potential new treatments called biologic therapies. The targets of these biological therapies are directed toward the B cell depletion, interference in the co-stimulation signals and the blockade of cytokines. Therapies using anti-CD20 monoclonal antibodies have shown satisfactory results especially in patients with SLE refractory to conventional treatment. The biological therapies provide encouraging results that represent a possible option in the treatment of refractory patients as well as a potential therapy in the future management of SLE.

(Rev Med Chile 2010; 138: 881-887).

Key words: Antibodies, monoclonal; B cell activating factor; Biological therapy; Lupus erythematosus, systemic; Rituximab.

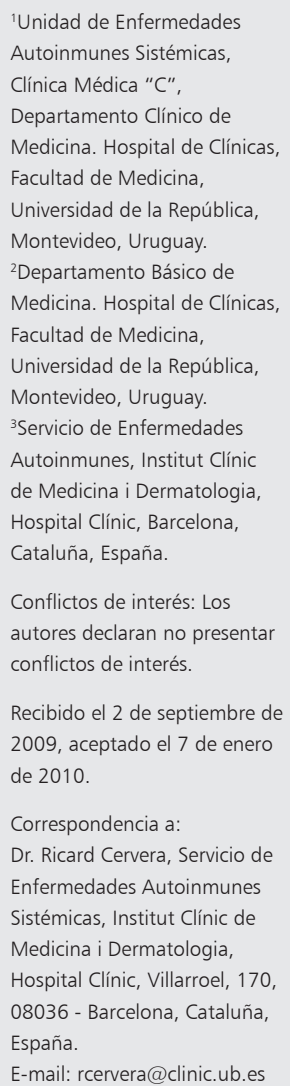

L a utilización de hidroxicloroquina, glucocorticoides y agentes inmunodepresores ha contribuido a mejorar de forma significativa el pronóstico de pacientes con lupus eritematoso sistémico (LES). Sin embargo, los efectos indeseables de los glucocorticoides así como el mayor riesgo de infecciones y neoplasias relacionado con los inmunodepresores, han motivado la búsqueda de nuevas terapéuticas que permitan una inmunodepresión más específica y con menor toxicidad. A partir de la experiencia generada en enfermedades como artritis reumatoide y enfermedad de Crohn entre otras, algunos de estos tratamientos comenzaron a ser utilizados en enfermedades autoinmunes sistémicas (EAS) como el LES, el síndrome antifosfolipídico y las vasculitis sistémicas primarias. Estas nuevas alternativas terapéuticas, denominadas de forma genérica terapias biológicas, han mostrado resultados alentadores. Esta revisión se centra en los resultados obtenidos por estos agentes en el tratamiento del LES.
Los mecanismos implicados en la inmunopatogenia del LES sobre los que las terapias biológicas pueden actuar son: 1) el número y/o función de los linfocitos B; 2) en las señales de coestimulación en la cooperación entre linfocitos T y B y 3 ) en los mediadores inmunitarios representados por las citocinas.

\section{Terapias biológicas dirigidas contra los linfocitos B}

Las terapias biológicas pueden actuar a nivel de los linfocitos $\mathrm{B}$ dando lugar a la disminución de su número o en la modulación de su función, actuando sobre diferentes dianas moleculares mediante: 1) anticuerpos anti-CD20, 2) anticuerpos anti-CD22, 3) inhibidores de los estimuladores de linfocitos B y 4) inductores de tolerancia o tolerágenos específicos de linfocitos B. 


\section{Anticuerpos anti-CD20 (Rituximab)}

La molécula CD20 es una glicoproteína transmembrana de $35 \mathrm{kDa}$ localizada en la superficie celular de los linfocitos B. Se expresa en las etapas madurativas pre- $\mathrm{B}$, linfocito inmaduro, maduro y memoria, estando ausente en la fase inicial de célula progenitora, linfocito pro-B y en la fase final de diferenciación hacia célula plasmática. $\mathrm{Al}$ actuar sobre CD20 se produce una disminución del número de linfocitos $\mathrm{B}$, bloqueando su diferenciación hacia células plasmáticas y disminuyendo la producción de autoanticuerpos. Rituximab (RTX) es un anticuerpo monoclonal (AcMo) quimérico dirigido contra CD20, resultante de la combinación de una región constante kappa de inmunoglobulina (Ig) G1 humana y una región variable de origen murino ${ }^{1}$. Los mecanismos que conducen a la disminución de linfocitos B mediados por RTX son la activación del complemento, la citotoxicidad mediada por anticuerpos y la inducción de apoptosis ${ }^{2}$. El RTX además induce una disminución de moléculas coestimuladoras CD40-ligando en linfocitos $\mathrm{T}$, en los marcadores del fenotipo de activación y en el número de células natural killer, incrementando además en número y función los linfocitos $\mathrm{T}$ reguladores ${ }^{2,3}$. En el caso del LES, el RTX ha sido utilizado en pacientes pediátricos, nefropatía lúpica, lupus neuropsiquiátrico, trombocitopenia y anemia hemolítica autoinmune entre otras ${ }^{4}$.

Las formas de administración del RTX en el LES son básicamente dos. El protocolo de mayor utilización ha sido la pauta indicada en el tratamiento del linfoma, $375 \mathrm{mg} / \mathrm{m}^{2}$ intravenoso de manera semanal durante 4 semanas consecutivas. La otra pauta es la aprobada para artritis reumatoide, administrando dos infusiones de $1.000 \mathrm{mg}$ de RTX intravenoso separadas una de otra de dos semanas ${ }^{1,5}$. Una vez administrado, la depleción de linfocitos B se mantiene durante 6 a 12 meses, aunque la recuperación de sus niveles puede verse a partir del $4^{\circ}$ mes $^{4,6,7}$.

Los primeros resultados disponibles de la utilización del RTX en el LES fueron los publicados por Leandro y cols $^{8,9}$. El ensayo valoró la eficacia del RTX en el tratamiento de la nefropatía lúpica activa resistente al tratamiento convencional. Fueron incluidos 24 pacientes con una media de seguimiento de 23 meses. El período de depleción de linfocitos B (linfocitos CD $19<5 / \mu l$ ) se mantuvo entre 3 y 11 meses. En esta serie, los 23 pacientes que lograron una depleción linfocitaria significativa (mayor al 99\%) presentaron mejoría. Los episodios de actividad del LES comenzaron una vez que los linfocitos B empezaron a ser detectables en sangre periférica9 .

En otro ensayo que incluyó 22 pacientes con nefropatía lúpica en actividad refractarios al tratamiento convencional, la adición de RTX al tratamiento inmunodepresor, logró en el 90\% una reducción significativa en los niveles de actividad y una disminución significativa de la proteinuria a 90 días de realizado el tratamiento ${ }^{3}$.

Las recaídas pueden ocurrir a 6 meses de restablecido el nivel de linfocitos B, como ocurrió en 7 de 11 pacientes tratados con $\mathrm{RTX}^{10}$. Si bien los resultados en la nefropatía lúpica se muestran alentadores, es importante destacar que los estudios presentan un corto intervalo de tiempo de seguimiento, lo que dificulta evaluar la persistencia de su beneficio en la evolución de la enfermedad.

Gürcan y cols ${ }^{1}$ analizaron 17 estudios en los que el RTX se utilizó en pacientes con LES refractario. En 159 pacientes de un total de 208 (79\%), se logró una mejoría clínica definida por descensos significativos en las escalas de actividad, mejoría de la función renal y disminución en la dosis de prednisona.

Existen menos datos del tratamiento con RTX en pacientes con lupus neuropsiquiátrico. Su utilización mostró mejoría en pacientes con delirium, disfunción cognitiva, psicosis y convulsiones ${ }^{11}$. En casos de anemia hemolítica autoinmune y púrpura trombocitopénica autoinmune en pacientes con LES, los resultados disponibles proceden de casos aislados, obteniendo buena respuesta terapéutica en la mayoría de ellos ${ }^{4}$. En general, la frecuencia de respuesta terapéutica al RTX en pacientes con afección cutánea, mucosa, articular, hematológica o del sistema nervioso central, se obtiene en un rango de 89 a $94 \%$ de los casos ${ }^{12}$.

Recientemente se ha dado a conocer los resultados de dos ensayos multicéntricos en pacientes con LES para valorar la eficacia y seguridad del RTX como parte del tratamiento. El primero, denominado EXPLORER, incluyó 250 pacientes con LES moderado a grave en tratamiento con un fármaco inmunodepresor (http://clinicaltrials.gov/ct2/ show/NCT00137969). El segundo, denominado LUNAR, incluyó 250 pacientes con nefritis lúpica clase III o IV, evaluando la eficacia del tratamiento al añadir RTX al micofenolato de mofetilo (http:// clinicaltrials.gov/ct2/show/study/NCT00282347). 
Ninguno de los dos ensayos alcanzó sus objetivos primarios, pero ello probablemente se debió a problemas metodológicos en sus diseños.

El RTX puede tener efecto en los marcadores inmunológicos de actividad lúpica como los anticuerpos anti-DNA y la fracción C3 del complemento. En pacientes que muestran una depleción linfocitaria B más sostenida, los niveles plasmáticos de estos marcadores presentan una mayor tendencia a disminuir y aumentar respectivamente 9 . Sin embargo, estas modificaciones no han sido descritas por otros autores ${ }^{3,13}$. El panel de los otros auto-anticuerpos se ve afectado de diferente forma con el tratamiento con RTX. En un estudio, los títulos de anticuerpos anti-DNA descendieron hasta $60 \%$, sin embargo, los niveles de anticuerpos como los anti-Ro (SSA), anti-La (SSB), anti-RNP y anti-Sm, se mantuvieron estables a pesar del tratamiento ${ }^{14}$.

En un tercio de los pacientes tratados con RTX se ha demostrado el desarrollo de anticuerpos anti-quiméricos humanos (HACA, human antichimeric antibodies), hecho asociado a una menor eficiencia en la depleción de linfocitos $\mathrm{B}^{6,15}$. En el estudio de Looney y cols ${ }^{13}, 11$ de 17 pacientes $(64,7 \%)$ desarrollaron HACA, 6 de ellos a títulos altos (mayores de $100 \mathrm{ng} / \mathrm{ml}$ ). La presencia de los HACA puede disminuir la eficacia del tratamiento con RTX o condicionar la necesidad de repetir su administración en el futuro ${ }^{13}$ o bien utilizar un AcMo anti-CD20 completamente humanizado como el ocrelizumab ${ }^{16}$.

La susceptibilidad a infecciones en pacientes con EAS tratados con RTX es difícil de establecer, ya que la infección podría estar relacionada con la enfermedad de base o con los tratamientos inmunodepresores utilizados. En un estudio, tras el tratamiento con RTX se produjo una tendencia a la disminución global del nivel de inmunoglobulinas que sin embargo, se mantuvo dentro de los rangos de la normalidad y no se asoció al desarrollo de infecciones graves ${ }^{14}$. La respuesta tras la vacunación anti-neumococo y anti-toxoide tetánico en pacientes con LES tratados con RTX, ha mostrado defectos importantes en la producción de anticuerpos específicos, por lo que se recomienda (de ser posible) realizar la vacunación antes de la administración del RTX ${ }^{7}$. Se comunicaron casos de reactivación de infecciones latentes por citomegalovirus, virus Epstein-Barr y virus de la hepatitis $\mathrm{B}^{7}$. Recientemente se publicaron 2 casos de leucoencefalopatía multifocal progresiva (LMP) en pacientes con LES tratados con RTX. La misma se produjo tras la utilización del tratamiento inmunodepresor y varios ciclos de RTX. Si bien éste podría incrementar el desarrollo de LMP, el riesgo absoluto de que ésta se produzca es muy bajo ${ }^{17,18}$.

Basados en los resultados de una revisión sistemática reciente, el RTX podría ser considerado como la terapia biológica de primera línea en pacientes con LES refractarios al tratamiento estándar ${ }^{5}$. De todas maneras existen muchas cuestiones sin resolver acerca de cuál es la dosis adecuada, si debe administrarse asociado a prednisona o a inmunodepresores, si puede ser utilizado en pacientes con formas menos graves de LES, si es posible evitar el desarrollo de HACA con la administración de inmunodepresores o si pueden los HACA cambiar la farmacocinética y duración del efecto del RTX. Tampoco existe información acerca de la utilidad de retratar a los pacientes una vez que los linfocitos B retornan a sus niveles normales, ni de si es posible combinar el RTX con otras terapias biológicas ${ }^{19,20}$.

Otro anticuerpo anti-CD20 es el ocrelizumab, un AcMo con la particularidad de ser un anticuerpo humanizado, menos inmunogénico y potencialmente mejor tolerado ${ }^{21}$. Lamentablemente, el ensayo clínico en pacientes con LES debió suspenderse debido a la aparición de infecciones oportunistas.

\section{Anticuerpos anti-CD22 (Epratuzumab)}

La molécula CD22 es una glicoproteína de 135 $\mathrm{kDa}$ con expresión restringida a los linfocitos $\mathrm{B}$. Está presente en el citoplasma de linfocitos pro-B y pre-B y se expresa en la superficie celular sólo en las etapas maduras del linfocito $\mathrm{B}$, desapareciendo en la diferenciación hacia células plasmáticas. El epratuzumab es un AcMo IgG1 dirigido contra CD22 con la particularidad de ser un anticuerpo humanizado, reduciendo potencialmente su antigenicidad. Su efecto inmunomodulador se cumple desencadenando señales inhibitorias sin inducir una destrucción específica de los linfocitos B.

En un estudio abierto que incluyó 14 pacientes con LES, el epratuzumab disminuyó los índices de actividad un 50\% y los linfocitos B un 35\%, sin disminuir los niveles de anticuerpos anti-DNA y sin detectar anticuerpos anti-epratuzumab ${ }^{22,23}$.

El hecho de que el epratuzumab y el RTX tengan mecanismos de acción diferentes, abre una puerta a una potencial asociación sinérgica entre ambos, 
teniendo como antecedente inmediato su combinación en el tratamiento de pacientes con linfomas ${ }^{24}$.

\section{Bloqueadores del Estimulador de Linfocitos B-BLyS (B lymphocyte stimulator) (Belimumab y Atacicept)}

Una de las moléculas involucradas en la diferenciación y proliferación linfocitaria es el estimulador de linfocitos B (B lymphocyte stimulator) BLyS, citocina perteneciente a la superfamilia del factor de necrosis tumoral- $\alpha$, secretado principalmente por monocitos-macrófagos y células dendríticas. En pacientes con LES existe una elevación de BLyS relacionada directamente con los incrementos de anticuerpos anti-DNA nativo ${ }^{25}$.

El belimumab es un AcMo IgG1 humanizado, capaz de unirse con alta afinidad a la forma soluble de BLyS en el plasma, evitando su unión a los receptores específicos en los linfocitos B. En un ensayo clínico en fase I que incluyó 70 pacientes, el belimumab fue capaz de reducir de forma significativa el número de linfocitos $\mathrm{B}$ pero sin inducir cambios en las escalas de actividad entre el grupo tratado y el control ${ }^{26}$. Recientemente se ha comunicado los resultados de dos ensayos en fase III que han demostrado su eficacia en pacientes con LES en actividad moderada y grave.

Otra vía para bloquear el efecto de BLyS es mediante el atacicept, una proteína de fusión recombinante formada por el dominio extracelular de la molécula transmembrane activator and calcium modulator ligand interactor (TACI) (receptor de BLyS) y el dominio constante de una IgG1 humana. Tiene la propiedad de bloquear al mismo tiempo el efecto de BLyS y el de la molécula denominada a proliferation inducing ligand (APRIL), de similares características estructurales y funcionales que BLyS. Con este doble efecto de bloqueo los ensayos clínicos en fase Ib con la administración subcutánea e intravenosa de atacicept, han demostrado reducciones dependientes de la dosis tanto en los niveles de inmunoglobulinas como en el número de linfocitos $\mathrm{B}^{27,28}$.

\section{Inductores de tolerancia de linfocitos B: Tolerágeno LJP 394 (Abetimus)}

Los tolerágenos de linfocitos B son moléculas capaces de inducir tolerancia al reaccionar de forma cruzada con receptores de linfocitos B autorreactivos, lo que conduce a la anergia o a la apoptosis.

El LJP 394 o abetimus, es una molécula formada por cuatro brazos de oligonucleótidos de doble cadena unidos a una plataforma de polietilenglicol no inmunogénica. Su utilización en diferentes estudios clínicos en pacientes con nefropatía lúpica (con presencia de títulos de anticuerpos anti-DNA mayores de $15 \mathrm{U} / \mathrm{ml}$ ) mostraron una rápida y sostenida reducción de los niveles de anticuerpos anti-DNA y un aumento en los niveles de la fracción C3 del complemento sin encontrar diferencias significativas en la actividad del LES y siendo ineficaz en prevenir la aparición de recaídas renales ${ }^{29-31}$.

\section{Modulación de la cooperación linfocito B-linfocito T: Inhibición de la señal de coestimulación}

La presentación antigénica incompleta por ausencia de coestimulación o segunda señal, sería el mecanismo responsable de desencadenar anergia y/o apoptosis, "silenciando" los linfocitos T e impidiendo la amplificación de la respuesta celular y humoral.

\section{Inhibidor del coestímulo CD40: CD40L}

La señal de coestímulo CD40: CD40L, se produce por la interacción entre CD40 (ubicado en la superficie de los linfocitos B) y CD40 Ligando (CD40L) (ubicado sobre la superficie de los linfocitos T). Ello activa los linfocitos B hacia su diferenciación y proliferación clonal. En pacientes con LES en actividad, se ha detectado un aumento en la expresión de CD40L en los linfocitos T así como un aumento de CD40 soluble en plasma ${ }^{32}$.

El IDEC-131 es un AcMo dirigido contra CD40L (conocido también como anti-CD154). En un ensayo clínico, se utilizó sin demostrar beneficio significativo, sufriendo un accidente cerebrovascular uno de los pacientes incluido en el grupo tratado ${ }^{32}$. El BG-9588 es un anticuerpo humanizado anti-CD40L utilizado en un ensayo clínico en fase II que incluyó 28 pacientes. Si bien mejoró los parámetros de actividad del LES, dicho estudio debió ser interrumpido por la aparición de un infarto agudo de miocardio en dos de los pacientes tratados ${ }^{33}$. Las trombosis asociadas a la administración de anticuerpos anti-CD40L 
podrían ser causadas por la reacción de dichos anticuerpos contra la molécula CD40L expresada sobre la superficie de las plaquetas activadas, induciendo su agregación y favoreciendo los fenómenos trombóticos ${ }^{34}$.

\section{Inhibidor del coestímulo B7-CD28}

Otra señal de coestimulación relevante es la establecida entre la molécula B7 (B7.1 y B7.2), presente en la superficie de células presentadoras de antígeno y linfocitos $\mathrm{B}$ activados y la molécula CD28, expresada de forma constitutiva en la superficie del linfocitos T. Una vez activado el linfocito T, se produce la expresión de un receptor adicional, homólogo de CD28, denominado CTLA-4 (o $\mathrm{CD} 152$ ) que tiene la propiedad de unirse a B7 con mayor avidez, induciendo una potente señal inhibitoria en el linfocito T volviéndolo menos sensible a estímulos externos (mecanismo esencial para limitar la proliferación de linfocitos $\mathrm{T}$ activados).

El CTLA-4-Ig o Abatacept, es una proteína de fusión formada por la unión de CTLA-4 y el fragmento constante de una IgG1 humana, comportándose como un receptor soluble de moléculas B7. El Abatacept es capaz de unirse a B7 con una afinidad muy superior a la que posee CD28, bloqueando la señal de coestímulo B7-CD28, evitando la activación linfocitaria. Los estudios con Abatacept en modelos murinos de LES han mostrados buenos resultados. En humanos se dispone de evidencia en artritis reumatoide, con resultados alentadores y potencialmente trasladables al tratamiento del $\mathrm{LES}^{35,36}$.

\section{Terapias anticitocinas}

\section{Bloqueadores del Factor de Necrosis Tumoral- $\alpha$ (TNF- $\alpha)$}

Los ensayos clínicos que utilizaron anti-TNF- $\alpha$ en el tratamiento del LES son escasos, abiertos y con inclusión de un número bajo de pacientes. En dos ensayos clínicos se administró infliximab encontrando una reducción de la actividad clínica pero objetivando incrementos en los anticuerpos anti-DNA nativos y anticuerpos anticardiolipina $^{37,38}$. Este fenómeno (descrito también con el etanercept y adalimumab) da muestra de su capacidad para inducir la producción de autoanticuerpos y enfermedades secundarias a su administración tales como LES y vasculitis cutáneas ${ }^{39}$.
Bloqueadores de interleucinas (IL)

Bloqueo de la IL-10. En pacientes con LES se realizó un estudio abierto con el AcMo murino IgG1 anti-IL10, objetivando una mejoría en las lesiones cutáneas y articulares pero sin disminución en los niveles de anticuerpos anti-DNA nativo. En todos los casos se detectaron anticuerpos contra el AcMo administrado ${ }^{40}$.

Bloqueo de la IL-1. El bloqueo de la actividad biológica de la IL-1 se puede lograr mediante Anakinra, un análogo recombinante antagonista de su receptor. En un único ensayo se trataron 4 pacientes con afectación articular logrando mejoría clínica y serológica, reapareciendo los síntomas en dos pacientes luego de la suspensión del tratamiento ${ }^{41}$.

Bloqueo de la IL-6. La utilización de anticuerpos anti-IL-6 en el tratamiento del LES, cuenta con suficiente evidencia en modelos murinos ${ }^{42}$. La reciente aprobación del uso del anticuerpo antiIL-6 (tocilizumab) en el tratamiento de la artritis reumatoide, convierte a este agente en una atractiva propuesta terapéutica para pacientes con $\mathrm{LES}^{43}$.

\section{Otras terapias biológicas}

Los tolerágenos específicos de anticuerpos anti-DNA nativo como el Edratide y el anticuerpo monoclonal dirigido contra la fracción C5 del complemento (Eculizumab) han sido utilizados en modelos animales con resultados alentadores ${ }^{44,45}$.

\section{Conclusiones}

Para considerar la indicación de una terapia biológica en pacientes con LES es necesario, en primer lugar, haber agotado la posibilidad del tratamiento inmunodepresor convencional. En caso de ser refractario al mismo, presentar intolerancia, efectos adversos graves o estar frente a una situación crítica con riesgo vital inminente, será necesario valorar de forma individual cada situación así como la relación riesgo-beneficio antes de optar por su utilización. Queda por resolver el interrogante de si la combinación de diferentes terapias biológicas potenciaría sus beneficios sin olvidar que sus efectos secundarios y toxicidad a largo plazo aún deben ser evaluados. Probablemente en el futuro podamos disponer de estudios controlados y aleatorizados, que respon- 
dan preguntas como cuáles son las dosis correctas, la duración del tratamiento, las combinaciones más apropiadas con los inmunodepresores y sobre todo qué subgrupos de pacientes con LES podrían beneficiarse en mayor medida de la utilización de estas terapias biológicas.

Agradecimientos: El Dr. Ernesto Cairoli realizó su estadía de formación en el Servicio de Enfermedades Autoinmunes del Hospital Clínic con el apoyo de la Agencia Española de Cooperación Internacional para el Desarrollo (AECID) (Gobierno de España) y del Programa de Investigaciones Biomédicas (PRO.IN.BIO), Facultad de Medicina, Universidad de la República, Uruguay.

\section{Referencias}

1. Gürcan H, Keskin D, Stern J, Nitzberg M, Shekhani H, Ahmed A. A review of the current use of rituximab in autoimmune diseases. International Immunopharmacology 2009; 9: 10-25.

2. Liossis S, Sfikakis P. Rituximab-induced B cell depletion in autoimmune diseases: potencial effects on $\mathrm{T}$ cells. Clin Immunol 2008; 127: 280-5.

3. Vigna-Pérez M, Hernández-Castro B, Paredes-Saharopulos O, Portales-Pérez D, Barabda L, Abud-Mendoza C, et al. Clinical and immunological effects of rituximab in patients with lupus nephritis refractory to conventional therapy: a pilot study. Arth Res Ther 2006; 8: 83-91.

4. García-Carrasco M, Jiménez-Hernández M, Escárcega R, Mendoza-Pinto C, Galarza-Maldonado C, SandovalCruz M, et al. Use of rituximab in patients with lupus systemic erythematosus: an update. Autoimmun Rev 2009; 8: 343-8.

5. Ramos-Casals M, Brito-Zerón P, Muñoz S, Soto MJ; BIOGEAS Study Group. A systematic review of the off-label use of biological therapies in systemic autoimmune diseases. Medicine (Baltimore) 2008: 87: 345-64.

6. Albert D, Dunham J, Khan S, Stansberry J, Kolasinski $\mathrm{S}$, Tsai D, et al. Variability in the biological response to anti-CD20 B cell depletion in systemic lupus erythematosus. Ann Rheum Dis 2008; 67: 1724-31.

7. Looney R, Srinivasan R, Calabrese L. The effects of rituximab on immunocompetency in patients with autoimmune disease. Arthritis Rheum 2008; 58: 5-14.

8. Leandro M, Edwards J, Cambridge G, Ehrenstein M, Isenberg D. An open study of B lymphocyte depletion in systemic lupus erythematosus. Arthritis Rhem 2002; 46: 2673-7.

9. Leandro M, Cambridge G, Edwards J, Ehrenstein M,
Isenberg D. B-cell depletion in the treatment of patients with systemic lupus erythematosus: a longitudinal analysis of 24 patients. Rheumatology 2005; 44: 1542-5.

10. Smith K, Jones R, Burns S, Jayne D. Long-term comparison of rituximab treatment for refractory systemic lupus erythematosus and vasculitis: remission, relapse and retreatment. Arthritis Rheum 2006; 54: 2970-82.

11. Tokunaga M, Saito K, Kawabata D, Imura Y, Fujii T, Nakayamada S, et al. Efficacy of rituximab (anti-CD20) for refractory systemic lupus erythematosus involving the central nervous system. Ann Rheum Dis 2007; 66: 470-5.

12. Hughes G. Rituximab in lupus and beyond: the state of the art. Lupus 2009; 18: 639-44.

13. Looney J, Anolik H, Campbell D, Felgar R, Young F, Arend L, et al. B cell depletion as a novel treatment for systemic lupus erythematosus. A phase I/II doseescalation trial of rituximab. Arthritis Rheum 2004; 50: 2580-9.

14. Cornec D, Avouac J, Youinou P, Saraux A. Critical analysis of rituximab - induced serological changes in connective tissue diseases. Autoimmun Rev 2009; 8: 515-9.

15. Thatayatikom A, White AJ. Rituximab: a promising therapy in systemic lupus erythematosus. Autoimmun Rev 2006; 5: 18-24.

16. Tahir H, Rohrer J, Bhatia A, Wegener W, Isenberg D. Humanized anti-CD20 monoclonal antibody in the treatment of severe resistant systemic lupus erythematosus in a patient with antibodies against rituximab. Rheumatology (Oxford) 2005; 44: 561-2.

17. Harris HE. Progressive multifocal leucoencephalopathy in a patient with systemic lupus erythematosus treated with rituximab. Rheumatology (Oxford) 2008; 47: 2245.

18. Carson K, Evens A, Richey E, Habermann T, Focosi D, Seymour J, et al. Progressive multifocal leukoencephalopathy after rituximab therapy in HIV-negative patients : a report of 57 cases from the research on adverse drug events and reports project. Blood 2009; 113: 4834-40.

19. Eisenberg R. Riruximab in lupus. Arthritis Res Ther 2003; 5: 157-9.

20. Espinosa G, Cervera R. Papel de los fármacos biológicos en el tratamiento de las enfermedades autoinmunitarias. Med Clin (Barcelona) 2007; 128: 456-7.

21. Ding C, Foote S, Jones G. B-cell-targeted therapy for systemic lupus erythematosus. An update. Biodrugs 2008; 22: 239-49.

22. Dörner T, Kaufmann J, Wegener W, Teoh N, Goldenberg D, Burmester G. Inicial clinical trial of epratuzumab (humanized anti-CD22 antibody) for immunotherapy 
of systemic lupus erythematosus. Arthritis Res Ther 2006; 8: R74.

23. Eisenberg R. Targeting B cells in systemic lupus erythematosus: not just déjà vu all over again. Arthritis Res Ther 2006; 8: 108-9.

24. Espinosa G, Ortego N, Sánchez-Román J. Terapias biológicas en el lupus eritematoso sistémico. "Avances en lupus eritematoso sistémico”. En Cervera R, JiménezAlonso J, editores. Marge Médica Books; 2008. p. 187203.

25. Stohl W, Metyas S, Tan Sm, Cheema G, Oamar B, Xu D, et al. B lymphocyte stimulator overexpression in patients with systemic lupus erythematosus. Longitudinal observations. Arthritis Rheum 2003; 48: 3475-86.

26. Furie R, Stohl W, Ginzler E, Becker M, Mishra N, Chatham W, et al. Biologic activity and safety of belimumab, a neutralizing anti-B-lymphocyte stimulator (BLyS) monoclonal antibody: a phase I trial in patients with systemic lupus erythematosus. Arthritis Res Ther 2008; 10: R109.

27. Dall'Era M, Chakravarty E, Wallace D, Genovese M, Weisman M, Kavanaugh A, et al. Reduced B lymphocyte and immunoglobulin levels after atacicept treatment in patients with systemic lupus erythematosus. Results of a multicenter, phase Ib, double-blind, placebo-controlled, dose-escalating trial. Arthritis Rheum 2007; 56: 4142-50.

28. Pena-Rossi C, Nasonov E, Stanislav M, Yakusevich V, Ershova O, Lomareva N, et al. An exploratory doseescalating study investigating the safety, tolerability, pharmacokinetics and pharmacodynamics of intraveous atacicept in patients with systemic lupus erythematosus. Lupus 2009; 18: 547-55.

29. Furie R, Cash J, Cronin M, Katz R, Weisman M, Aranow $\mathrm{C}$, et al. Treatment of systemic lupus erythematosus with LJP 394. J Rheumatol 2001; 28: 257-65.

30. Alarcón-Segovia D, Tumlin J, Furie R, Mckay J, Cardiel M, Strand V, et al. LJP 394 for the prevention of renal flare in patients with systemic lupus erythematosus: results from a randomized, double-blind, placebocontrolled study. Arthritis Rheum 2003; 48: 442-54.

31. Cardiel M, Tumlin J, Furie R, Wallace D, Joh T, Linnik M, LJP 394-90-09 investigator consortium. Abetimus sodium for renal flare in systemic lupus erythematosus : results of a randomized, controlled phase III trial. Arthritis Rheum 2008; 58: 2470-80.

32. Kalunian K, Davis J, Merrill J, Totoritis M, Wofsy D. IDEC-131 Lupus Study Group. Treatment of systemic lupus erythematosus by inhibition of $\mathrm{T}$ cel costimulation with anti-CD154. A randomized, double-blind, placebo-controlled trial. Arthritis Rheum 2002; 46: 3251-8.
33. Boumpas D, Furie R, Manzi S, Illei G, Wallace D, Balow J, et al. A short course of BG9588 (anti-CD40 ligand antibdy) improves serologic activity and decreases hematuria in patients with proliferative lupus glomerulonephritis. Arthritis Rheum 2003; 48: 719-27.

34. Kawai T, Andrews D, Colvin R, Sachs D, Cosimi A. Thromboembolic complications after treatment with monoclonal antibody against CD40 ligand. Nat Med 2000; 6: 114

35. Davidson A, Diamond B, Wofsy D, Daikh D. Block and tackle: CTLA-4Ig takes on lupus. Lupus 2005; 14: $197-$ 203.

36. Westhovens R, Verschueren P. Translating co-stimulation blockade into clinical practice. Arthritis Res Ther 2008; 10 (Suppl 1): S4.

37. Aringer M, Graninger W, Steiner G, Smolen J. Safety and efficacy of tumor necrosis factor blockade in systemic lupus erythematosus. An open-label study. Arthritis Rheum 2004; 50: 3161-9.

38. Aringer M, Steiner G, Graninger W, Höfler E, Steiner C, Smolen J. Effects of short-term infliximab therapy on autoantibodies in systemic lupus erythematosus. Arthritis Rheum 2007; 56: 274-9.

39. Ramos-Casals M, Brito-Zerón P, Soto MJ, Cuadrado MJ, Khamashta M. Autoimmune diseases induced by TNFtargeted therapies. Best Pract Res Clin Rheumatol 2008; 22: 847-61.

40. Lorente L, Richaud-Patin Y, García-Padilla C, Claret E, Jakez-Ocampo J, Cardiel M, et al. Clinical and biologic effects of anti-interleukin-10 monoclonal antibody administration in systemic lupus erythematosus. Arthritis Rheum 2000; 43: 1790-800.

41. Ostendorf B, Iking-Konert C, Kurz K, Jung G, Sander O, Schneider M. Preliminary results of safety and efficacy of the interleukin 1 receptor antagonist anakinra in patients with severe lupus arthritis. Ann Rheum Dis 2005; 64: 630-3.

42. Tackey E, Lipsky P, Illei G. Rationale for interleukin-6 blockade in systemic lupus erythematosus. Lupus 2004; 13: 339-43.

43. Scheinecker C, Smolen J, Yasothan U, Stoll J, Kirkpatrick P. Tocilizumab. Nat Rev Drug Discovery 2009; 8: 273-4.

44. Elmann A, Sharabi A, Dayan M, Zinger H, Ophir R, Mozes E. Altered gene expression in mice with lupus treated with Edratide, a peptide that ameliorates the disease manifestations. Arthritis Rheum 2007; 56: 2371-81.

45. Rother R, Mojcik C, Mccroskery E. Inihibition of terminal complement: a novel therapeutic approach for the treatment of systemic lupus erythematosus. Lupus 2004; 13: $328-34$. 